\title{
Assessment of Probabilistic Methods for Mistuned Bladed Disk Vibration
}

\author{
Soo-Yeol Lee, Matthew P. Castanier $†$ and Christophe Pierre ${ }^{\ddagger}$ \\ Department of Mechanical Engineering \\ The University of Michigan, Ann Arbor, MI 48109-2125
}

\begin{abstract}
In this paper, the accuracy and efficiency of various probabilistic methods are assessed for the vibration analysis of a mistuned bladed disk system with small, random bladeto-blade differences. A quantity of primary engineering interest is examined, namely the maximum resonant response amplitude of any blade in the assembly over a given frequency range. For the purpose of comparing the probabilistic methods, the response amplitude of a specific blade at a given resonant frequency is also considered. Initially, the following methods are applied to analyze the effects of small blade stiffness uncertainties for a lumpedparameter model of a 29-blade rotor: (1) a first-order reliability method (FORM), (2) a second-order reliability method (SORM), (3) an advanced mean value (AMV+) method, (4) a response surface method (RSM) using a moving least squares approach, and (5) a radius-based importance sampling method. In general, these methods do well in predicting the response statistics of a given blade at a given frequency, at least in limited ranges of uncertainties. However, all of these reliability-based methods fail to capture the statistics of the maximum resonant response across the blade assembly, regardless of the range of uncertainties. To circumvent this shortcoming, an accelerated Monte Carlo simulation (MCS) approach is also considered, which involves a combination of a small-sample MCS and a Weibull probability distribution fit. This method is found to predict the statistics of the largest resonant blade response over the blade assembly with great accuracy and efficiency. It is thus better suited to the analysis of bladed disk response statistics than the class of reliability-based probabilistic methods.
\end{abstract}

\section{Introduction}

Structural system always has some parameter uncertainties due to manufacturing tolerances, material A property variations, in-service degradation, etc. This variability consequently produces a variability in the dynamic response, which may be significant for systems that are sensitive to uncertainties, such as nominally periodic structures. ${ }^{1,2}$ For bladed disk structures, which are found in jet engines and other turbomachinery, the vibratory response of blades is known to feature high (sometimes extreme) sensitivity to the small, random blade-to-blade differences that are referred to as blade mistuning. ${ }^{3-5}$ Since larger blade response means larger stress, fatigue, and possibly failure, it is critically important to be able to predict accurately the effects of uncertainties in blade properties on the vibratory response of bladed disk systems. Because blade mistuning is caused by manufacturing tolerances, it is a random quantity and a probabilistic approach is required.

For a mistuned bladed disk system such as the compressor stage shown in Fig. 1, the variable of interest is the maximum resonant response amplitude of any blade in the assembly over a frequency range of interest. Since mistuning is known only in a statistical sense for a population of manufactured bladed disks, and because additional mistuning is introduced by wear and damage during the operating life of the structure, it is important for the designer to predict the statistics of the maximum resonant blade response in terms of those of the blade mistuning. From such a probabilistic analysis, the high-percentile blade forced response can be obtained at the design stage (e.g., the 99.99th percentile of the largest resonant response, which

* Graduate Student Research Assistant, Student Member of AIAA

${ }^{\dagger}$ Associate Research Scientist, Senior Member of AIAA

$\ddagger$ Collegiate Professor, Senior Member of AIAA 
will be exceeded by only one out of every 10,000 bladed disks fabricated), which can then be used to avoid high-cycle fatigue and failure.

The objectives of this paper are (1) to apply various reliability-based methods for probabilistic analysis, all of which are extensively used in structural reliability and durability, to the vibratory blade response of a mistuned bladed disk system and (2) to investigate their performance and limitations. In the paper, a simple yet representative lumped-parameter model of the bladed disk in Fig. 1 is selected in order to assess the various probabilistic methods. The statistics of the maximum resonant blade response-the quantity of primary engineering interest-are considered, and so are the statistics of the response of a given blade at a given resonance. The methods examined include most probable point (MPP)-based methods, such as a first-order reliability method (FORM), a second-order reliability method (SORM), and an advanced mean value (AMV) method. The response surface method (RSM) using the moving least squares approach is also considered, and so is a radius-based importance sampling method. An alternative probabilistic approach that is specialized to bladed disk vibration is also examined, namely an accelerated Monte Carlo simulation. The method is applied and compared with the above reliability analysis methods.

Among reliability-based methods, FORM is the most widely used analytical approximation technique, in which the limit state surface is replaced by a hyperplane. ${ }^{6}$ Thus, the closer the limit state surface is to a hyperplane, the more accurate the result produced by FORM. However, if the limit state surface has a large curvature and is not far from the origin, the reliability estimated by FORM may deviate considerably from the true value. In order to address this deficiency and improve the accuracy of FORM, second-order methods have been developed..$^{7-9}$ The SORM approximates a limit state surface by a quadratic surface at the MPP, and it has been extensively utilized in structural safety problems. ${ }^{10,11}$ An advanced mean value (AMV) method has been proposed to improve the approximation of mean-value first-order (MVFO) method. ${ }^{12}$ The AMV method uses the MPP of the first-order approximated limit state surface, and compensates for the truncation error. It has been used in reliability analysis for fatigue life, ${ }^{13}$ in vibration power flow analysis, ${ }^{14}$ and in other applications. Various response surface construction methods have also been developed and most of them can be classified as global methods, using a single quadratic or cubic polynomial to represent the uncertainty space. Since global methods use a single polynomial, they can introduce large errors when approximating a nonlinear response surface. Thus, in order to describe the locally nonlinear behavior of the response, a moving least squares response surface construction method has been developed, which uses the compact supported weighting functions..$^{15,16}$ In addition, the radius-based importance sampling method has been proposed ${ }^{17}$ as a way to increase the accuracy of the FORM and the efficiency of the basic Monte Carlo technique. It is a sampling method based on the calculation of a reliability index ( $\beta$-sphere). Finally, in previous work by the authors, an accelerated Monte Carlo simulation method was proposed for estimating the statistics of the maximum forced response of a mistuned bladed disk. ${ }^{18}$

The original contributions of this paper can be summarized as follows. First, several representative reliability-based probabilistic methods are applied to the prediction of the statistics of the vibration response of mistuned bladed disk structures; this represents a new field of application for this class of techniques. Second, the performance of these methods is systematically assessed in terms of accuracy and efficiency, and their applicability and limitations are determined for bladed disk structures. Third, the effectiveness of these methods is also compared to that of traditional and accelerated Monte Carlo simulation methods that have been previously used for bladed disk structures.

The paper is organized as follows. The lumped-parameter bladed disk model is described first. Next, the reliability analysis methods used to calculate the mistuned bladed disk response statistics are presented. Then, the accelerated Monte Carlo simulation method, which is specialized to the bladed disk problem, is introduced. All these methods are subsequently applied to the mistuned bladed disk model, and their performance and accuracy are compared and discussed. Conclusions are given in the final section of the paper.

\section{Two-DOF-Per-Sector Model of a Turbomachinery Rotor}

Although bladed disks in turbomachinery rotors are typically represented by detailed finite element models, their dynamics can often be accurately described using alternate lumped-parameter models with only one or two degrees of freedom (DOF) per sector of the bladed disk. In this paper, the industrial compressor rotor with 29 blades shown in Fig. 1 is considered. This system was studied in Ref. 19, and it is modeled as having two DOF per sector, as shown in Fig. 2. 


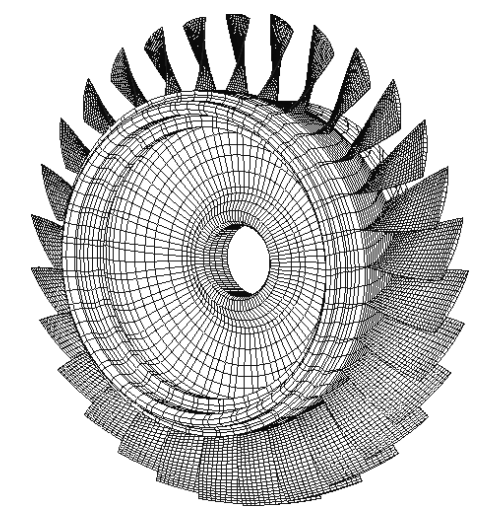

Figure 1. Finite element model of an industrial compressor rotor.

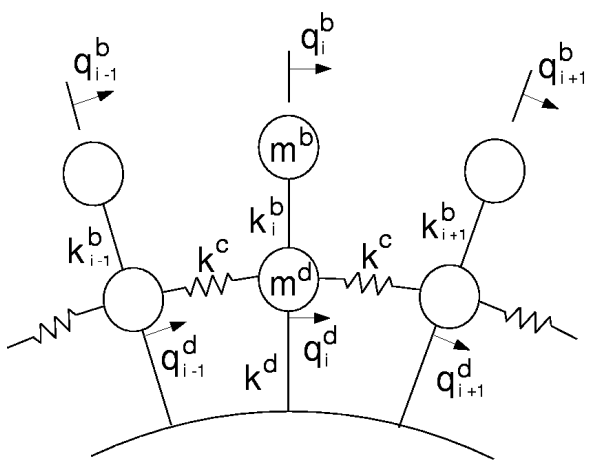

Figure 2. Two-DOF-per-sector model.

In this type of model, which has been used extensively in the literature (e.g., Refs. 20,21), one DOF represents the blade motion and the other DOF represents the disk motion, and the coupling between blades occurs through the disk. The blade and disk DOF have mass $m^{b}$ and $m^{d}$, respectively, and adjacent disk DOF are connected by coupling springs with stiffness $k^{c}$. Two stiffness elements, $k_{i}^{b}$ and $k^{d}$, represent the blade and disk stiffness, respectively, for the $i$ th sector. It is assumed that the structural uncertainties are accounted for in the expression of the mistuned blade-alone stiffness, as

$$
k_{i}^{b}=k_{o}^{b}\left(1+\delta_{i}\right) \quad i=1,2, \ldots, N_{b}
$$

where $k_{o}^{b}$ is the nominal (tuned) blade-alone stiffness, $\delta_{i}$ is the blade stiffness variation (that is, the mistuning for the $i$ th blade), and $N_{b}$ is the number of blades. For the analysis of the forced response of the two-DOFper-sector model to a harmonic engine order excitation, the mass and stiffness parameters are normalized with the nominal blade properties, resulting in a set of dimensionless parameters as

$$
\bar{m}=\frac{m^{d}}{m^{b}} \quad \bar{k}_{c}=\frac{k^{c}}{k_{o}^{b}} \quad \bar{k}_{d}=\frac{k^{d}}{k_{o}^{b}} \quad \omega_{o}^{b}=\sqrt{\frac{k_{o}^{b}}{m^{b}}}
$$

Then, assuming harmonic oscillations, the equation of motion for the two-DOF-per-sector lumped parameter model can be expressed by

$$
\left[-\omega^{2} \mathbf{M}+(1+j \eta) \mathbf{K}\right] \mathbf{q}=\mathbf{F}
$$

where $\eta$ is the structural damping factor. The mass matrix, $\mathbf{M}$, the stiffness matrix, $\mathbf{K}$, and the applied force vector, $\mathbf{F}$, can be explicitly written as

$$
\begin{array}{ccc}
\mathbf{M}=\left[\begin{array}{cc}
\operatorname{diag}(\bar{m}) & \mathbf{0} \\
\mathbf{0} & \mathbf{I}
\end{array}\right] & \mathbf{K}=\left[\begin{array}{cc}
\mathbf{K}_{D D} & -\mathbf{K}_{B B} \\
-\mathbf{K}_{B B} & \mathbf{K}_{B B}
\end{array}\right] \\
\mathbf{F}=\left[\begin{array}{c}
\mathbf{0} \\
\mathbf{f}_{B}
\end{array}\right] & \mathbf{q}=\left[\begin{array}{c}
\mathbf{q}_{D} \\
\mathbf{q}_{B}
\end{array}\right]
\end{array}
$$

The sub-matrices of the stiffness matrix $\mathbf{K}$ can be defined as

$$
\begin{aligned}
& \mathbf{K}_{D D}=\operatorname{circ}\left(1+2 \bar{k}_{c}+\bar{k}_{d},-\bar{k}_{c}, \cdots,-\bar{k}_{c}\right)+\operatorname{diag}\left(\delta_{i}\right) \\
& \mathbf{K}_{B B}=\mathbf{I}+\operatorname{diag}\left(\delta_{i}\right)
\end{aligned}
$$

where circ denotes a circulant matrix with the arguments being the first row, and diag denotes a diagonal matrix with the argument being the $i$ th diagonal element. And, the sub-matrices, $\mathbf{q}_{D}$ and $\mathbf{q}_{B}$, are for the degrees of freedom associated with the disk and the blade, respectively, and are written as

$$
\begin{aligned}
& \mathbf{q}_{D}=\left[\begin{array}{llll}
q_{1}^{d} & q_{2}^{d} & \cdots & q_{N_{b}}^{d}
\end{array}\right]^{T} \\
& \mathbf{q}_{B}=\left[\begin{array}{llll}
q_{1}^{b} & q_{2}^{b} & \cdots & q_{N_{b}}^{b}
\end{array}\right]^{T}
\end{aligned}
$$


The external force applied here is a harmonic excitation that differs only in phase from one blade to the next. This type of excitation, called engine order excitation, is due to the rotation of the bladed disk in the flow field. Thus, the sub-vector $\mathbf{f}_{\mathrm{B}}$ can be defined as

$$
\mathbf{f}_{B}=\frac{F}{m^{b}}\left[\begin{array}{llll}
1 & e^{j \phi_{2}} & \cdots & e^{j \phi_{N_{b}}}
\end{array}\right]
$$

where

$$
\phi_{i}=\frac{2 \pi C(i-1)}{N_{b}}
$$

and $C$ is the engine order of the excitation, and $N_{b}$ is the number of blades.

The parameters of the two-DOF-per-sector model of the industrial rotor in Fig. 1 were identified based on the geometric characteristics of the natural frequency curves in the veering region shown in Fig. 3, using a method developed in Ref. 22. This frequency versus nodal diameters plot characterizes the free vibration of the bladed disk, with the horizontal lines representing blade-dominated modes, the slanted lines representing disk-dominated modes, and the veering regions being indicative of significant disk-blade interactions, where large forced response sensitivity to mistuning occurs. Here the veering region of interest concerns the family of blade-dominated modes, which is characterized by a mixed second torsion/second flexural bending $(2 \mathrm{~T} / 2 \mathrm{~F})$ mode shape for the blades. The engine order of the excitation is $C=2$, thus exciting the bladed disk in the $2 \mathrm{~T} / 2 \mathrm{~F}$ veering region. The lumped-parameter model obtained from the veering fit has the parameters of $\bar{m}=374.20, \bar{k}_{c}=2054.89, \bar{k}_{d}=43.98$, and $\omega_{o}^{b}=1$. In this study, the physical quantity of interest is the maximum response amplitude of any blade across the frequency range of interest (here the $2 \mathrm{~T} / 2 \mathrm{~F}$ resonant range), for each mistuning pattern.

This maximum response amplitude is normalized by the resonant tuned response amplitude $q_{T}$, yielding an amplification factor due to blade mistuning, which can be expressed as

$$
g(\mathbf{x})=\max _{1 \leq i \leq N_{b}} \max _{\omega \in \Omega}\left|q_{i}^{b}(\mathbf{x} ; \omega)\right| / q_{T}
$$

where $\Omega=[0.95,1.05]$ and $\mathbf{x}$ is the vector of 29 random variables of blade stiffnesses $k_{i}$. The function $g(\mathbf{x})$ will be used as a performance function in the probabilistic vibration analysis of the mistuned bladed disk system. In Fig. 4, for a specific mistuning pattern of standard deviation 1 percent, the responses of the 29 mistuned blades are shown. The maximum blade response for the tuned and mistuned systems are also compared by examining the envelope of maximum amplitude of any blade versus excitation frequency. The physical quantity of interest is the largest blade response of the mistuned system in Fig. 4. Thus, in order to predict the statistics of the maximum resonant blade response in terms of those of the blade mistuning, various probabilistic methods are considered.

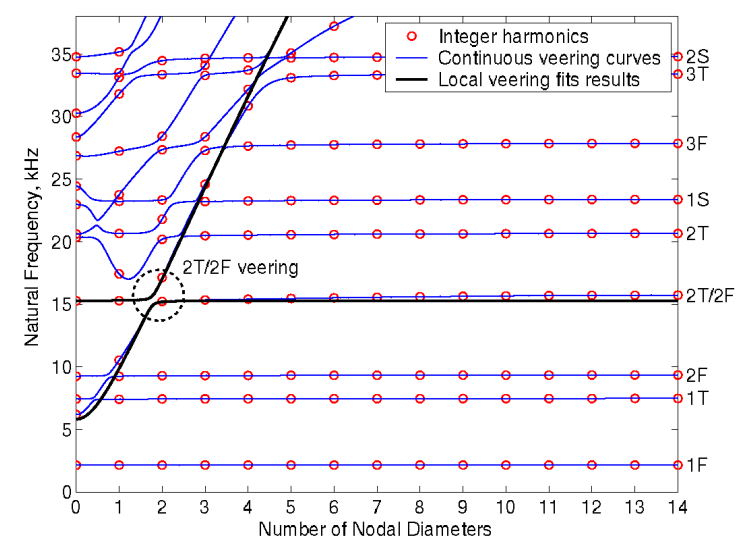

Figure 3. Local veering fit for $2 \mathrm{~T} / 2 \mathrm{~F}$ veering region of the industrial compressor rotor.

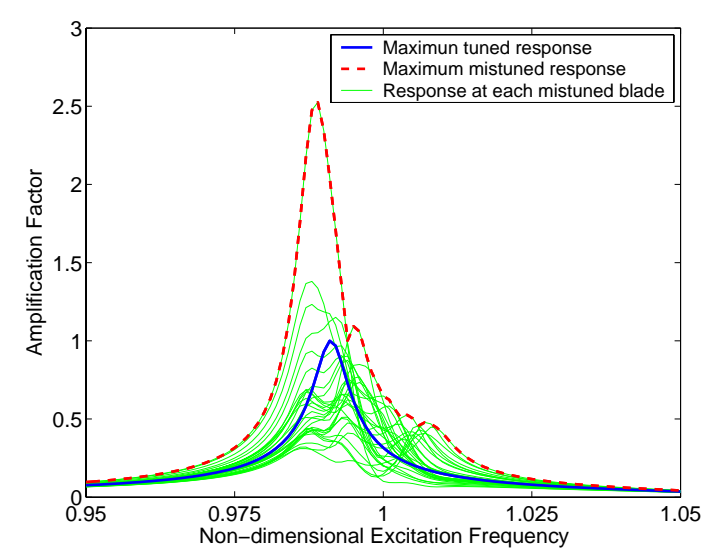

Figure 4. Maximum tuned response and maximum mistuned response. 


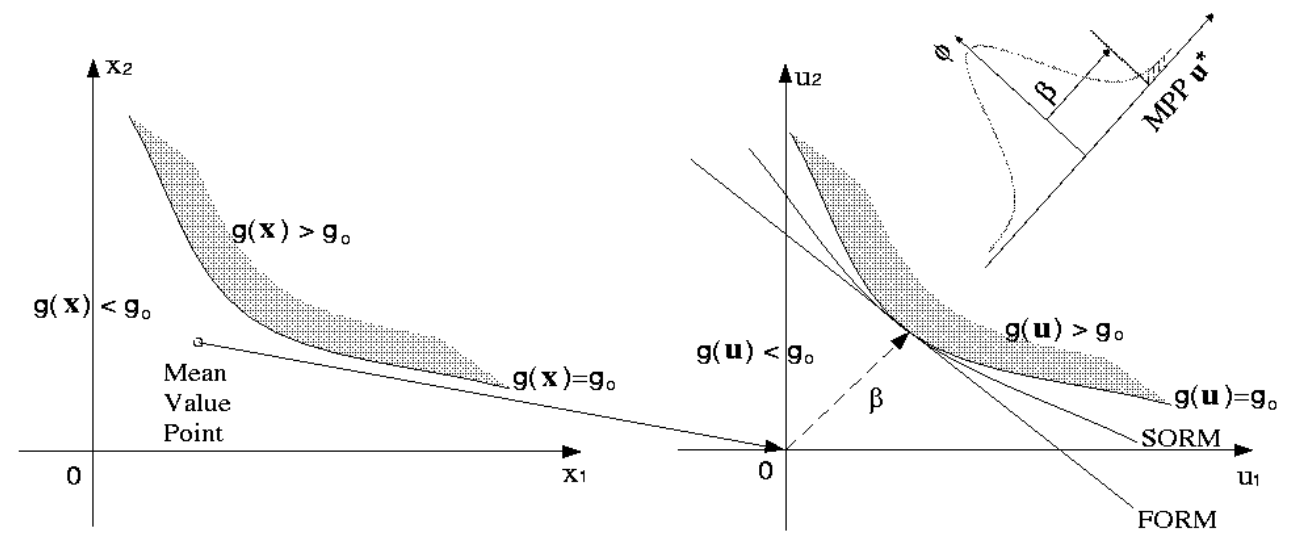

Figure 5. Most probable point and reliability index $\beta$.

\section{Reliability Analysis Methods}

For the structural reliability analysis, a performance function describing the structural behavior should first be defined. In order to access the various reliability analysis methods for two blade response cases, the performance function of Eq. (10) is given for the case of the maximum response amplitude of any blade over a given frequency range. And, for the case of the response amplitude of a specific blade at a given resonant frequency, the other performance function is defined as

$$
g(\mathbf{x})=\left|q_{i=1}^{b}(\mathbf{x} ; \omega=1)\right| / q_{T}
$$

Given the joint probability density function $f_{\mathbf{x}}(\mathbf{x})$ of the random variables $\mathbf{x}$, the probability of the structural response can be expressed as

$$
P\left[g(\mathbf{x}) \leq g_{o}\right]=\iint \cdots \int_{g(\mathbf{x}) \leq g_{o}} f_{\mathbf{x}}(\mathbf{x}) d \mathbf{x}
$$

It is a fundamental but difficult problem in structural reliability to compute Eq. (12). With an implicit performance function, the multi-dimensional numerical integration can be extremely time-consuming. To evaluate the integral in Eq. (12) efficiently, various reliability analysis methods have been developed. In this paper, these methods are to be examined in view of two blade responses, Eq. (10) and Eq. (11)

\section{A. First-Order Reliability Method (FORM)}

In order to make use of properties of a standardized normal space, a transformation is introduced to map the basic random variable $x_{i}$ to the standardized uncorrelated normal variable $u_{i}$ as shown in Fig. 5 , such that

$$
T: u_{i}=\Phi^{-1}\left[F_{x_{i}}\left(x_{i}\right)\right] \quad i=1,2, \ldots, n
$$

where $\Phi$ is a cumulative distribution function (CDF) of the standardized normal distribution and $F_{x_{i}}$ is the CDF of the basic random variable $x_{i}$. Hasofer and Lind defined the reliability index as the smallest distance from the origin to a point on the limit state surface in a standardized normal space. ${ }^{23}$ The search for the minimum distance can be formulated as the following constrained optimization problem.

$$
\beta=\min \|\mathbf{u}\| \quad \text { subject to } g(\mathbf{u})=g_{o}
$$

The corresponding point $\mathbf{u}^{*}$ is called the most probable point (MPP). When the basic random variables are jointly normally distributed and the limit state surface is a hyperplane, the probability, where $g$ is less than or equal to $g_{o}$, is

$$
P\left[g \leq g_{o}\right]=\Phi(\beta)
$$


If the limit state surface is close to a hyperplane and it is far away from the origin in a standardized normal space, Eq. (15) gives a good approximation in many reliability problems.

From another perspective, a first-order inverse reliability method, called a performance measure approach (PMA), has been proposed in the context of a design optimization. ${ }^{24}$ In this technique, with a specified target reliability index, the target probabilistic performance measure $g^{*}=F_{g}^{-1}\left(\Phi\left(\beta_{t}\right)\right)$ is calculated, where $F_{g}$ is a CDF of a performance function. It can be defined in the context of vibration of a bladed disk system as follows:

$$
g^{*}=\max g(\mathbf{u}) \quad \text { subject to }\|\mathbf{u}\|=\beta_{t}
$$

In later sections, when a specified percentile response is needed, this approach will be employed.

\section{B. Second-Order Reliability Method (SORM)}

To improve the accuracy of the result of FORM, second-order reliability methods have been developed. More detailed description about SORMs can be found in Ref. 6-9. In a SORM, a performance function $g(\mathbf{x})$ is expanded into the Taylor series around the most probable point (MPP) $\mathbf{u}^{*}$ up to the second order in a standardized normal space

$$
g(\mathbf{u}) \simeq \nabla g\left(\mathbf{u}^{*}\right)^{T}\left(\mathbf{u}-\mathbf{u}^{*}\right)+\frac{1}{2}\left(\mathbf{u}-\mathbf{u}^{*}\right)^{T} \mathbf{D}\left(\mathbf{u}^{*}\right)\left(\mathbf{u}-\mathbf{u}^{*}\right)
$$

where $\nabla g\left(\mathbf{u}^{*}\right)$ is the gradient vector at the MPP and $\mathbf{D}$ is the symmetric matrix of second-order partial derivative at $\mathbf{u}^{*}$.

$$
D_{i j}\left(\mathbf{u}^{*}\right)=\frac{\partial^{2} g\left(\mathbf{u}^{*}\right)}{\partial u_{i} \partial u_{j}}
$$

The probability with the second-order approximation of the performance function can be expressed with a FORM reliability index $\beta$ and the main curvatures $\kappa_{j}$ of the limit state surface at MPP, which are determined as the eigenvalues of the Hessian matrix. Breitung has derived an asymptotic expression with a one-term formula, which yields good approximations for large values of $\beta .{ }^{7}$ Tvedt has derived a three-term approximation to the probability content inside the approximating quadratic surface as follows: 6,8

$$
P\left[g \leq g_{o}\right] \approx 1-\left(A_{1}+A_{2}+A_{3}\right)
$$

where

$$
\begin{aligned}
& A_{1}=\Phi(-\beta) \prod_{j=1}^{n-1}\left(1-\beta \kappa_{j}\right)^{-\frac{1}{2}} \\
& A_{2}=[\beta \Phi(-\beta)-\phi(\beta)]\left\{\prod_{j=1}^{n-1}\left(1-\beta \kappa_{j}\right)^{-\frac{1}{2}}-\prod_{j=1}^{n-1}\left(1-(\beta+1) \kappa_{j}\right)^{-\frac{1}{2}}\right\} \\
& A_{3}=(\beta+1)[\beta \Phi(-\beta)-\phi(\beta)]\left\{\prod_{j=1}^{n-1}\left(1-\beta \kappa_{j}\right)^{-\frac{1}{2}}-\operatorname{Re}\left[\prod_{j=1}^{n-1}\left(1-(\beta+\sqrt{-1}) \kappa_{j}\right)^{-\frac{1}{2}}\right]\right\}
\end{aligned}
$$

where $\operatorname{Re}[\quad]$ denotes the real part. $\Phi$ and $\phi$ are a cumulative distribution function and a probability density function for the standardized normal distribution. In this study, Eq. (19) will be utilized for a second order approximated probability.

\section{Advanced Mean Value (AMV) Method}

The AMV method has been proposed in Ref. 12, based on the mean-value first-order (MVFO) method. The performance function $g(\mathbf{x})$ is expanded into the Taylor series about the mean $\mathbf{x}=\boldsymbol{\mu}$ : 


$$
\begin{aligned}
g(\mathbf{x}) & =g(\boldsymbol{\mu})+\sum_{i=1}^{n}\left(\frac{\partial g}{\partial x_{i}}\right)_{\mathbf{x}=\boldsymbol{\mu}}\left(x_{i}-\mu_{i}\right)+H(\mathbf{x}) \\
& =a_{o}+\sum_{i=1}^{n} a_{i} x_{i}+H(\mathbf{x}) \\
& =g_{1}(\mathbf{x})+H(\mathbf{x})
\end{aligned}
$$

where $g_{1}(\mathbf{x})$ represents the sum of the first-order terms and $H(\mathbf{x})$ represents the higher-order terms. Based on this explicit and linear $g_{1}$, the most probable point (MPP) $\mathbf{x}^{*}$ can be obtained, and then the truncation error is compensated with $H\left(\mathbf{x}^{*}\right)$ to minimize the error in probability estimate.

The AMV method is based on the fact that the MPP of $g_{1}(\mathbf{x})$ is generally close to the exact MPP of $g(\mathbf{x})$, and thus, by using the linear performance function $g_{1}(\mathbf{x})$ approximated by a Taylor series expansion in a random variable space, a reasonable cumulative probability can be obtained.

In order to increase the accuracy of the prediction of probability, an iteration process can be applied to the AMV method. There are two iteration processes. One is for a specified probability level, and the other is for a specified $g$ level. ${ }^{12}$ Both methods require iterations that involve the evaluation of the $g$ function and its derivatives until a converged solution is obtained. In this paper, the iterative advanced mean value $(\mathrm{AMV}+)$ method for a specified probability level is used.

\section{Response Surface Method}

In order to predict the response of a system with limited number of data, various response surface methods have been developed. One of them uses a surface constructed with a moving least squares (MLS) method, which is widely used in meshless methods. ${ }^{15,16}$ The amplification factor $g(\mathbf{x})$ can be approximated by MLS interpolant in the domain $\Omega$ by

$$
g^{h}(\mathbf{x})=\sum_{i=1}^{m} p_{i}(\mathbf{x}) a_{i}(\mathbf{x})=\mathbf{p}^{T}(\mathbf{x}) \mathbf{a}(\mathbf{x})
$$

where $m$ is the number of terms in the basis, $p_{i}(x)$ are monomial basis functions, and $a_{i}(x)$ are their coefficients, which are functions of the spatial coordinates $\mathbf{x}$. In this study, a linear basis in a 29-dimensional domain was employed. Thus,

$$
\mathbf{p}^{T}=\left[\begin{array}{lllll}
1 & x_{1} & x_{2} & \cdots & x_{29}
\end{array}\right], \quad m=30
$$

The coefficients $a_{i}(\mathbf{x})$ in Eq. (24) can be obtained at any point $\mathbf{x}$ by minimizing the following:

$$
\begin{aligned}
J & =\sum_{j}^{n} w\left(\mathbf{x}-\mathbf{x}_{j}\right)\left[\mathbf{p}^{T}\left(\mathbf{x}_{j}\right) \mathbf{a}(\mathbf{x})-g\left(\mathbf{x}_{j}\right)\right]^{2} \\
& =(\overline{\mathbf{P}} \mathbf{a}-\overline{\mathbf{g}})^{T} \mathbf{W}(\mathbf{x})(\overline{\mathbf{P}} \mathbf{a}-\overline{\mathbf{g}})
\end{aligned}
$$

where $n$ is the number of points in the domain of influence of $\mathbf{x}$ for which the weight function $w\left(\mathbf{x}-\mathbf{x}_{j}\right) \neq 0$, and $g\left(\mathbf{x}_{j}\right)$ is the value of the amplification factor evaluated at the sampling point $\mathbf{x}=\mathbf{x}_{j}$. The matrices $\overline{\mathbf{g}}$, $\overline{\mathbf{P}}$ and $\mathbf{W}$ are defined as follows:

$$
\begin{gathered}
\overline{\mathbf{g}}^{T}=\left[\begin{array}{llll}
g\left(\mathbf{x}_{1}\right) & g\left(\mathbf{x}_{2}\right) & \cdots & g\left(\mathbf{x}_{n}\right)
\end{array}\right] \\
\overline{\mathbf{P}}=\left[\begin{array}{cccc}
p_{1}\left(\mathbf{x}_{1}\right) & p_{2}\left(\mathbf{x}_{1}\right) & \cdots & p_{m}\left(\mathbf{x}_{1}\right) \\
p_{1}\left(\mathbf{x}_{2}\right) & p_{2}\left(\mathbf{x}_{2}\right) & \cdots & p_{m}\left(\mathbf{x}_{2}\right) \\
\vdots & \vdots & \ddots & \vdots \\
p_{1}\left(\mathbf{x}_{n}\right) & p_{2}\left(\mathbf{x}_{n}\right) & \cdots & p_{m}\left(\mathbf{x}_{n}\right)
\end{array}\right]
\end{gathered}
$$


and

$$
\mathbf{W}(\mathbf{x})=\left[\begin{array}{cccc}
w\left(\mathbf{x}-\mathbf{x}_{1}\right) & 0 & \cdots & 0 \\
0 & w\left(\mathbf{x}-\mathbf{x}_{2}\right) & \cdots & 0 \\
\vdots & \vdots & \ddots & \vdots \\
0 & 0 & \cdots & w\left(\mathbf{x}-\mathbf{x}_{n}\right)
\end{array}\right]
$$

The stationarity of $J$ in Eq. (26) with respect to $\mathbf{a}(\mathbf{x})$ leads to the following linear relations between $\mathbf{a}(\mathbf{x})$ and $\overline{\mathrm{g}}$ :

$$
\mathbf{A}(\mathbf{x}) \mathbf{a}(\mathbf{x})=\mathbf{B}(\mathbf{x}) \overline{\mathbf{g}}
$$

where the matrix $\mathbf{A}$ and $\mathbf{B}$ are given by

$$
\mathbf{A}=\overline{\mathbf{P}}^{T} \mathbf{W}(\mathbf{x}) \overline{\mathbf{P}} \quad \mathbf{B}=\overline{\mathbf{P}}^{T} \mathbf{W}(\mathbf{x})
$$

Thus, one has

$$
\mathbf{a}(\mathbf{x})=\mathbf{A}^{-1}(\mathbf{x}) \mathbf{B}(\mathbf{x}) \overline{\mathbf{g}}
$$

and the approximate response surface can be constructed as

$$
g^{h}(\mathbf{x})=\mathbf{p}^{T}(\mathbf{x}) \mathbf{a}(\mathbf{x})=\mathbf{p}^{T}(\mathbf{x}) \mathbf{A}^{-1}(\mathbf{x}) \mathbf{B}(\mathbf{x}) \overline{\mathbf{g}}
$$

With this constructed response surface, the reliability analysis methods described in the previous sections, as well as a Monte Carlo simulation (MCS) method, can be applied for a probabilistic analysis. In this paper, a MCS method is employed with the constructed response surface. It should be noted that the accuracy of the constructed response surface depends on the number and the location of sampling points to construct a response surface.

\section{E. Radius-Based Importance Sampling Method}

This method is appropriate when a percentile value of the response is of interest, not for the entire cumulative distribution function $(\mathrm{CDF})$ of the performance function. This technique can be utilized with the transformation with Eq. (13) and the reliability index of Eq. (14).

As shown in Fig. 6, the sampling points located inside the hypersphere ( $\beta$-sphere) with radius $\beta$ are in the region of $g \leq$ $g_{o}$. Thus, it is not needed to calculate the performance function for these points in $D_{1}$. Only the points that are sampled outside the hypersphere $\left(D_{2}\right)$ are used to calculate the performance function values. The probability of being in the domain $D_{1}$ can be expressed as

$$
P_{1}=P\left[\sum_{i=1}^{n} u_{i}^{2} \leq \beta^{2}\right]=\chi_{n}^{2}\left(\beta^{2}\right)
$$

where $\chi_{n}^{2}\left(\beta^{2}\right)$ is the chi-squared distribution with $n$ degrees of freedom (equals the dimension of the random vector $\mathbf{x}$ ). Then, the probability of $P\left[g \leq g_{o}\right]$ can be estimated by

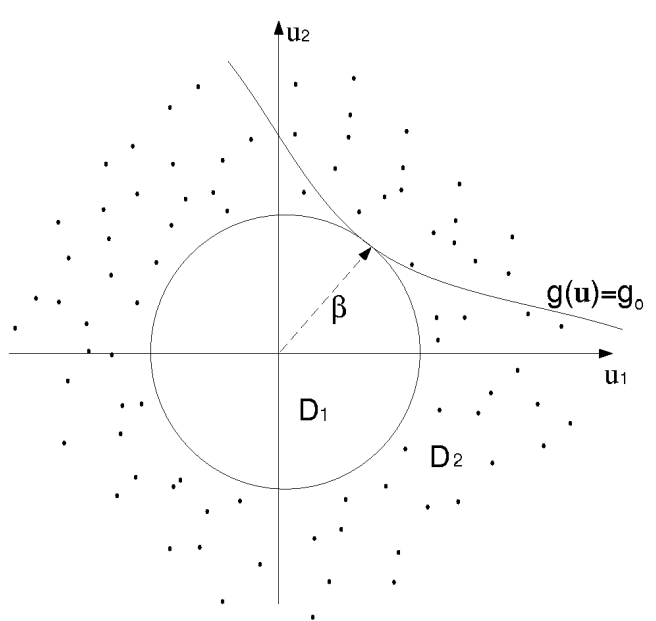

Figure 6. Radius-based importance sampling.

$$
P\left[g \leq g_{o}\right]=P_{1}+\frac{\left(1-P_{1}\right)}{N} \sum_{i=1}^{N} I\left[g\left(\mathbf{u}_{i}\right)\right]
$$

where $N$ is the number of sampling points in $D_{2}$ and $I\left[g\left(\mathbf{u}_{i}\right)\right]$ is as follows:

$$
I\left[g\left(\mathbf{u}_{i}\right)\right]= \begin{cases}0 & \text { if } g(\mathbf{u})>g_{o} \\ 1 & \text { if } g(\mathbf{u}) \leq g_{o}\end{cases}
$$

The efficiency of this method is known to be reduced as the number of random variables increases. This will be illustrated with numerical results in a later section. 


\section{Accelerated Monte Carlo Simulation Method}

A previous study by the authors demonstrated that the maximum response statistics of a bladed disk system with mistuning tends to a Weibull distribution and that the Weibull distribution parameters may be estimated from relatively few Monte Carlo (MC) realizations. ${ }^{18}$ This specialized probabilistic method, developed for a mistuned bladed disk system and called an accelerated Monte Carlo simulation method, is briefly described here.

It is known that the distribution of the maximum of $\mathrm{N}$ independently and identically distributed random trials approaches one of three asymptotes as $\mathrm{N}$ becomes large. For a bladed disk system, the amplification factor has a finite lower bound of zero and has a possible upper bound, even though this may not be specifically known. This non-zero upper bound suggests that the corresponding extremal distribution will converge to the Gumbel's type III asymptotic form. ${ }^{25}$ The type III asymptotic form, known as the threeparameter Weibull distribution, has a cumulative distribution function (CDF) of the form

$$
F(z)=\exp \left[-\left(\frac{\lambda-z}{\delta}\right)^{\eta}\right]
$$

where $\lambda, \delta$, and $\eta$ are known as the location, scale, and shape parameters, respectively. In this study, the location parameter, $\lambda$, is approximated by the theoretical maximum amplification factor, ${ }^{26}$ which was determined to be

$$
\lambda=\frac{1}{2}\left(1+\sqrt{N_{b}}\right)
$$

where $N_{b}$ is the number of the blades. Using this approximation for $\lambda$, the other two parameters can be systematically estimated from very few Monte Carlo realizations (e.g., 50 realizations). By manipulating Eq. (36), a linear relationship can be retrieved as

$$
\ln (\lambda-z)=\frac{1}{\eta} \ln (-\ln F)+\ln (\delta)
$$

Once the data has been cast into this form, a least squares linear regression may be used to estimate the other parameters, $\eta$ and $\delta$. Then, the three-parameter Weibull distribution can be constructed, which approximates the maximum response statistics of a mistuned bladed disk system. ${ }^{18,19}$

\section{Numerical Results}

To examine the applicability of the various probabilistic methods to a mistuned bladed disk system, two blade response cases are considered: one for the maximum resonant response amplitude of any blade in the assembly over a frequency range of interest, which is the quantity of primary engineering interest; and one

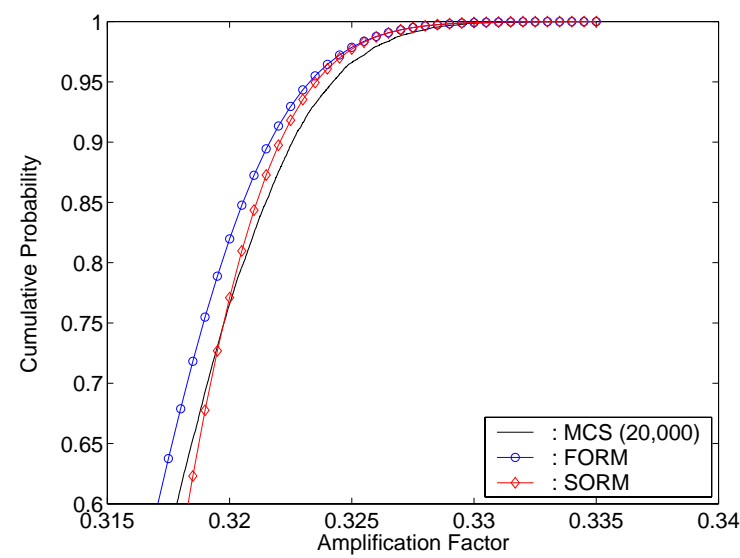

Figure 7. FORM/SORM for a fixed frequency and a specific blade $(\mathrm{COV}=0.05 \%)$.

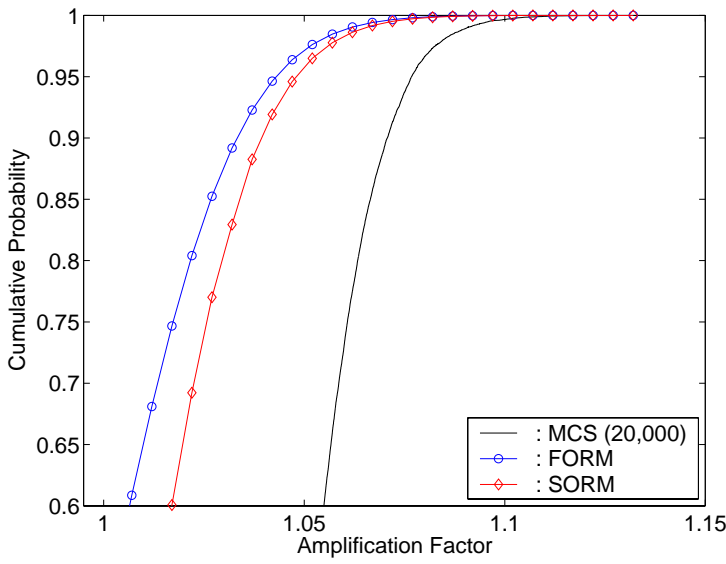

Figure 8. FORM/SORM for any frequency and any blade $(\mathrm{COV}=0.05 \%)$. 
for the response of a specific blade at a fixed frequency, which is for the comparison of the performance of the probabilistic methods. And, it is assumed that the 29-blade stiffnesses are the random variables with normal or uniform distributions.

First, a first-order reliability method (FORM) and a second-order reliability method (SORM) are employed for probabilistic vibration analysis of a bladed disk system with mistuning having normal distribution and a coefficient of variation $(\mathrm{COV})$ of $0.05 \%$, where the $\mathrm{COV}$ is defined as the ratio of the standard deviation to the mean value. The small COV value of $0.05 \%$ is chosen to compare the reliability analysis methods for two blade response cases of a mistuned bladed disk system excluding the effect of the range of uncertainty.

Figure 7 shows the cumulative probabilities for a specific blade at a fixed frequency, which are from a Monte Carlo simulation (MCS) with 20,000 realizations as well as from the FORM and the SORM. Both methods provide good approximations relative to the MCS, and the SORM shows more accurate result. In Fig. 8, the FORM and the SORM are applied to the maximum response of any blade for a frequency sweep in a bladed disk sys-

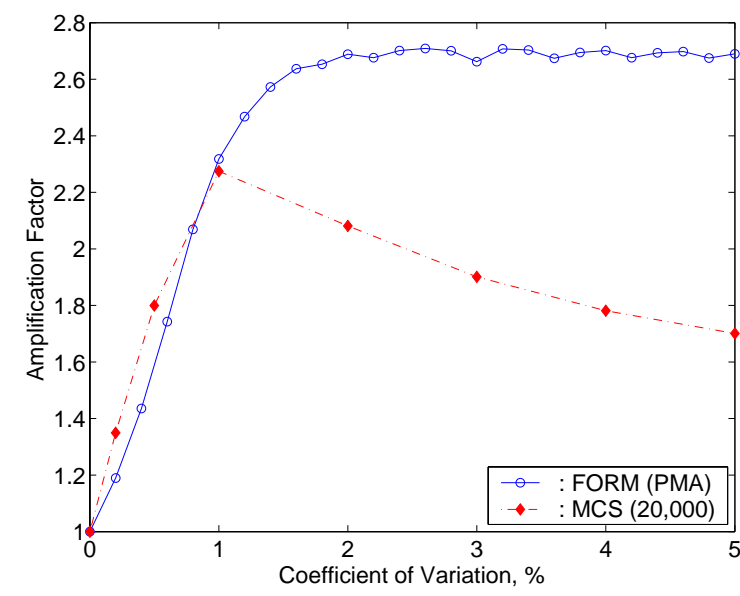

Figure 9. 95th percentile amplification factor for various $\mathrm{COV}$ values. tem. The cumulative probabilities from the FORM and the SORM show the difference from that from the MCS with 20,000 realizations in Fig. 8. The comparison of Fig. 7 and Fig. 8 implies that the case of maximum response amplitude of any blade over a frequency range of interest is more nonlinear, whereas the case of the response of a specific blade at a fixed frequency can approximately be assumed to behave monotonically in this limited range. The more relevant discussion can be made with the probability results from the AMV method.

In Fig. 8, the high percentile responses of the FORM and the SORM are close to that of the MCS. Thus, in Figs. 9-11, the high percentile responses of any blade for a frequency sweep are more investigated. For a specified percentile response with respect to some COV values, a first-order inverse reliability method, which is also called a performance measure approach (PMA), is utilized. Figure 9 shows the 95th percentile amplification factor of the maximum response with respect to the COV of the random variables (mistuning strength). The 95th percentile is defined as the magnitude of the response that is expected to be exceeded by only $5 \%$ of the maximum forced response. In Fig. 9, the result of the FORM is close to that of the MCS up to the COV of $1 \%$. However, above the COV of $1 \%$, there is a large difference between the FORM and the MCS results. To investigate this phenomenon more deeply, the MCS was performed at some specific distances

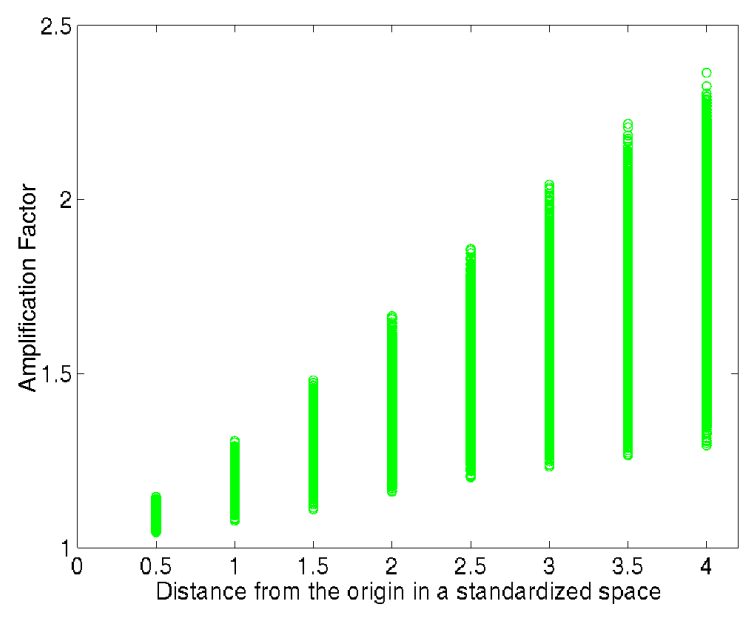

Figure 10. Amplification factor in a standardized normal space for $\mathrm{COV}=1 \%$.

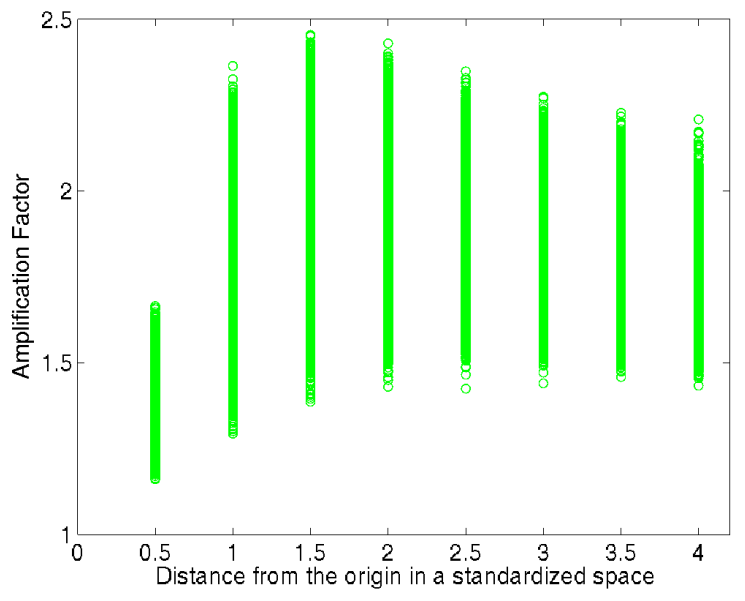

Figure 11. Amplification factor in a standardized normal space for $\mathrm{COV}=4 \%$. 


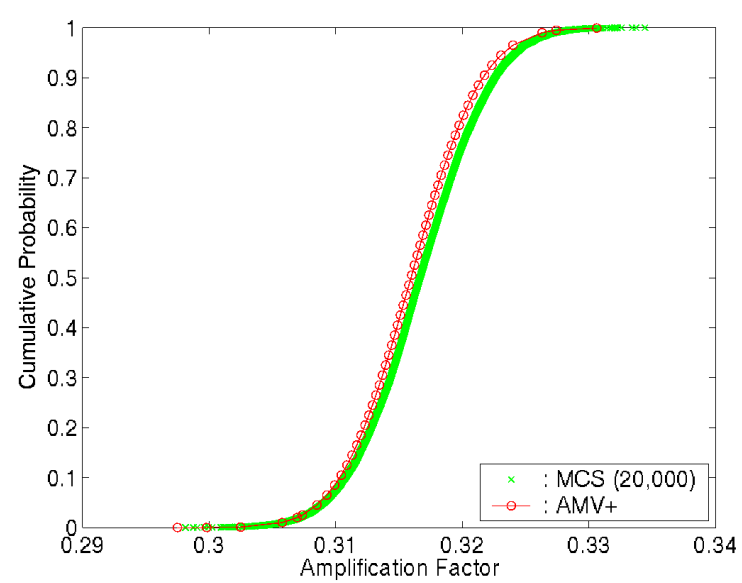

Figure 12. AMV method for a fixed frequency and a specific blade $(\mathrm{COV}=0.05 \%)$.

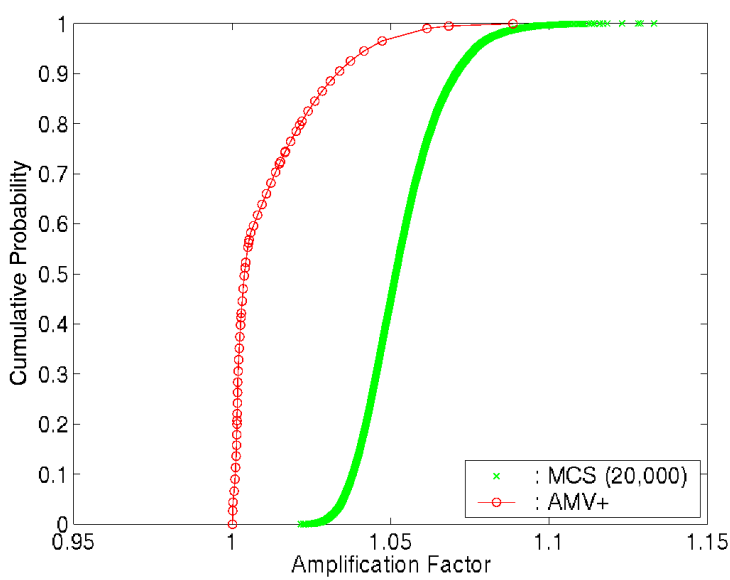

Figure 13. AMV method for any frequency and any blade $(\mathrm{COV}=0.05 \%)$.

in a standardized normal space for certain COV values. The cases of COV of $1 \%$ and $4 \%$ are shown in Fig. 10 and Fig. 11. These figures cannot directly show the degree of nonlinearity of the maximum response of any blade over a frequency sweep, but they can help to explain why the FORM provides high percentile response results that are close to those from the MCS in the limited range shown in Fig. 9. Up to the COV of $1 \%$, the largest value at each specific distance from the origin shows monotonic behavior and the possible highest value is far from the origin over a standardized normal space as shown in Fig. 10. However, above the $\mathrm{COV}$ of $1 \%$, the largest value at each specific distance from the origin shows nonmonotonic behavior, and the possible highest value is not far from the origin any more, as shown in Fig. 11. Thus, a prediction of the high percentile response, such as a 95th percentile response, with the FORM can be close to that of the MCS only up to the COV of $1 \%$. However, generally speaking, the FORM or the SORM cannot predict a probability of the maximum forced response of any blade for a frequency sweep.

Next, an advanced mean value $(\mathrm{AMV}+)$ method is employed for two blade response cases of a bladed disk system with mistuning having normal distributions. Figure 12 depicts the cumulative probabilities obtained from the MCS with 20,000 realizations and from the AMV+ method for a specific blade at a fixed frequency. Excellent agreement is seen for the cumulative probabilities. In Fig. 13, a cumulative probability obtained from the Monte Carlo results is compared with that calculated with the $\mathrm{AMV}+$ method for the maximum response of any blade for a frequency sweep. It shows that there is a significant difference between a result of the MCS with 20,000 realizations and that of the $\mathrm{AMV}+$ method.

The AMV method has been proposed such that it can estimate the probability by assuming multiple limitstates generated by a concave- or a convex-like performance function. ${ }^{12}$ Thus, the AMV method can handle a monotonic case and the nonlinear cases such as a concave-

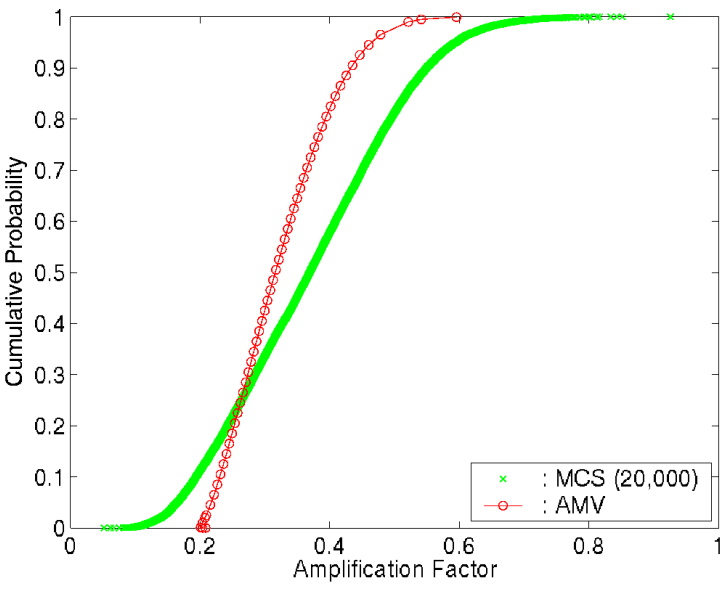

Figure 14. AMV method for a fixed frequency and a specific blade $(\mathrm{COV}=1 \%)$. or a convex-like function. However, when it is applied to a highly nonlinear performance function, it cannot give an accurate result as shown in Fig. 13 . Figure 14 shows the comparison of the cumulative probabilities between the MCS with 20,000 realizations and the AMV method for a specific blade at a fixed frequency at the COV of $1 \%$. In this case, at some probability levels, the AMV+ method shows a convergence problem. Thus, Fig. 14 shows the cumulative probability of the AMV method without iteration process. When the range of uncertainties (mistuning strength) is limited to the COV of $0.05 \%$, the prediction of the AMV+ method is excellent for a specific blade at a fixed frequency as in Fig. 12. However, as the range of uncertainties is expanded to the COV of $1 \%$, there is a 


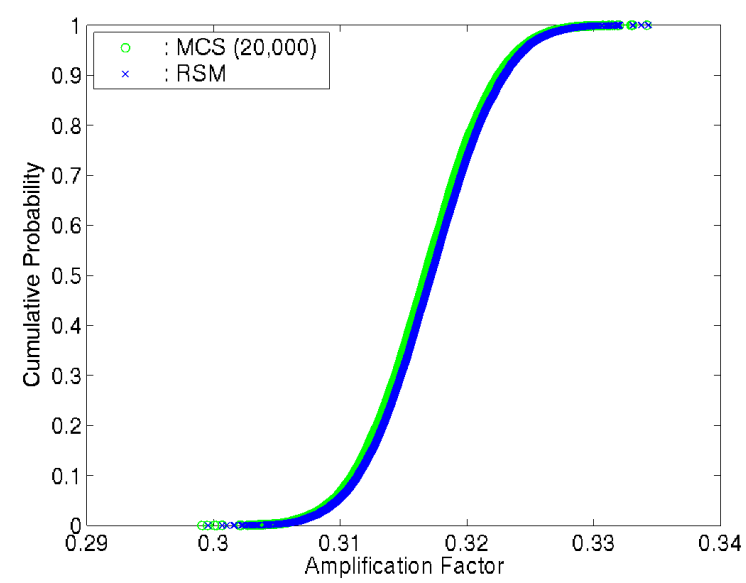

Figure 15. RSM for a fixed frequency and a specific blade $(\mathrm{COV}=0.05 \%)$.

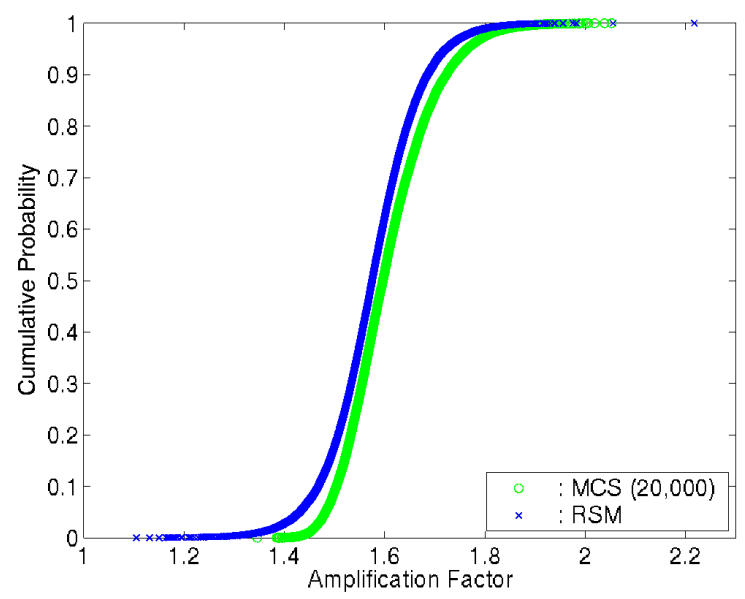

Figure 17. RSM for any frequency and any blade $(\mathrm{COV}=4 \%)$.

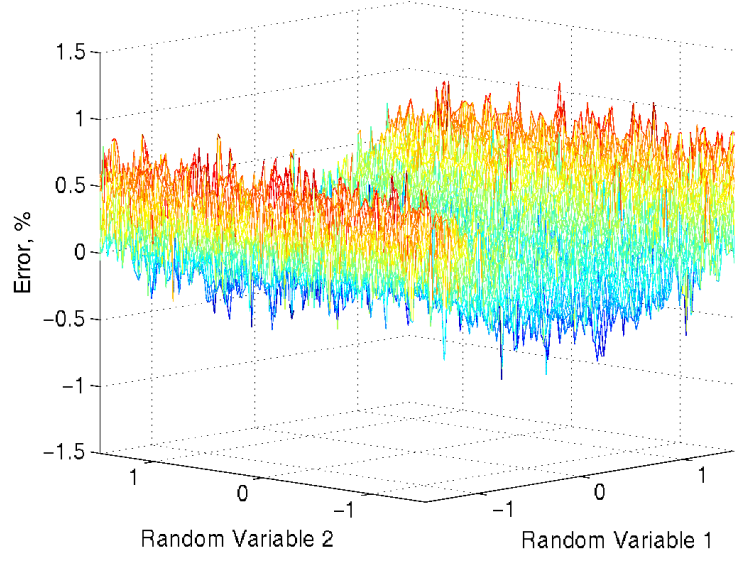

Figure 16. Orthogonal projection of error of RSM (fixed frequency and specific blade / $\mathrm{COV}=0.05 \%)$.

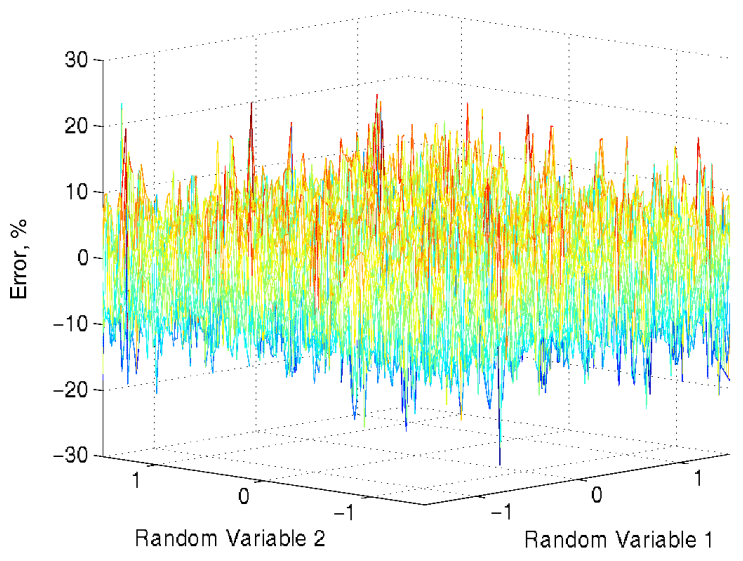

Figure 18. Orthogonal projection of error of RSM (any frequency and any blade $/ \mathrm{COV}=4 \%$ ).

significant difference between the Monte Carlo result and the result of the AMV+ method, even in the case of a specific blade at a fixed frequency.

Next, a response surface method (RSM) using a moving least squares (MLS) approach is employed for two blade response cases of a bladed disk system with mistuning having uniform distributions. To construct the response surface, 500 sampling points are chosen by a Latin Hypercube design. Then, in order to determine the statistical characteristics for a constructed response surface, the Monte Carlo simulation with 20,000 realizations is done. To distinguish the MCS with the lumped parameter model and the MCS with a constructed response surface, the latter is specified as RSM in Fig. 15 and Fig. 17. Figure 15 depicts the cumulative probabilities obtained from the MCS with 20,000 realizations and from the RSM for a specific blade at a fixed frequency. In order to confirm the agreement of the cumulative probabilities in Fig. 15, the errors between the MCS and the RSM are orthogonally projected onto the space of 2 random variables as in Fig. 16. The maximum error is $1.37 \%$, and it is seen that the RSM effectively describes the actual structural response surface for the response amplitude of a specific blade at a fixed frequency. As for the case of the maximum response amplitude of any blade for a frequency sweep, Fig. 17 shows the comparison of the cumulative probabilities between the MCS with 20,000 realizations and the RSM. The prediction of the probabilities with the RSM seems to be agreeable with that of the MCS in Fig. 17, but it turned out that the agreement is just coincidence of both probabilities. When the errors between the MCS and the RSM are 


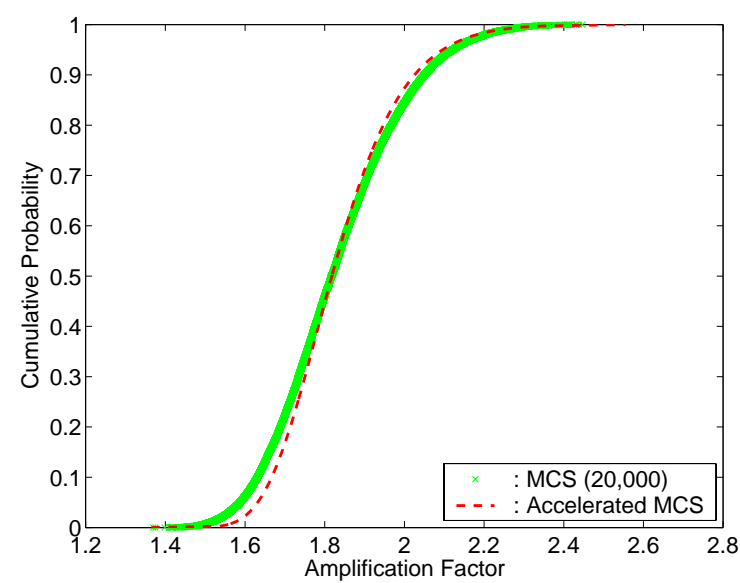

Figure 19. Accelerated MCS (any frequency and any blade, $\mathrm{COV}=1 \%$ ).

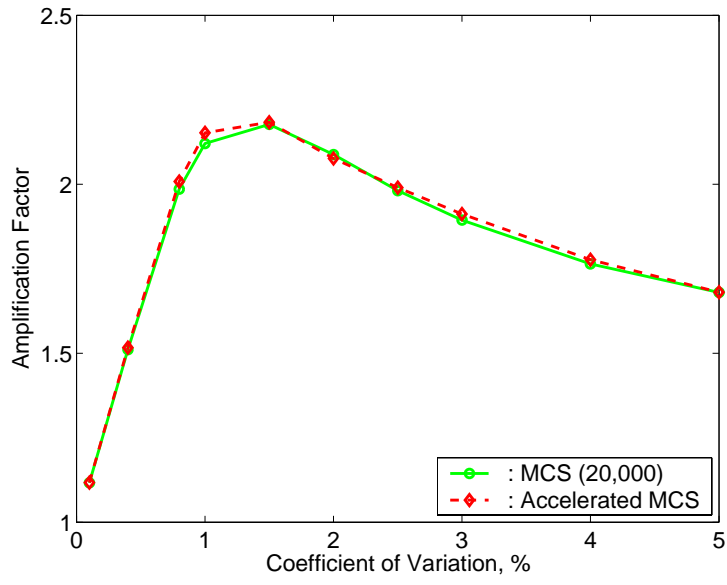

Figure 20. 95th percentile amplification factor for various COV values (any frequency and any blade).

orthogonally projected onto the space of 2 random variables in Fig. 18, the maximum error reaches up to 29.15\%. That means the response surface constructed with 500 sampling points cannot describe the actual structural response for the case of the maximum response amplitude of any blade for a frequency sweep. Thus, with the constructed response surface with 500 sampling points, the maximum response cannot be identified accurately for a given mistuning pattern. The RSM results also show that the response surface of the maximum response amplitude of any blade for a frequency sweep has greater nonlinearity, such that the response surface cannot be constructed well with 500 sampling points.

Next, the applicability of a radius-based importance sampling method is considered. For a case where a high percentile response of $99.9 \%$ is of interest, a radius of a sphere in Fig. 6 can be defined to be $\beta=3$. Then, in order to compare the efficiency of the MCS with 20,000 realizations, the sampling points of the MCS in $D_{1}$ are counted. There are only two such points out of 20,000 sampling points. This is predictable from the characteristics of the chi-squared distribution $\chi_{29}^{2}\left(\beta^{2}\right)$. Thus, the efficiency of this method is no more than that of the MCS for a case of 29 random variables. However, this radius-based importance sampling method will show better efficiency as the number of variables is reduced.

Finally, the accelerated MCS method is employed for a bladed disk system with mistuning having uniform distribution. The Weibull distribution was constructed by estimating its parameters based on data obtained from 50 MC realizations. In Fig. 19, the cumulative probability obtained from the MCS with 20,000 realizations is compared with that calculated with the accelerated MCS for the maximum response of any blade for a frequency sweep at the COV of $1 \%$. Figure 20 shows the comparison of the 95th percentile amplification factors between the MCS with 20,000 realizations and the accelerated MCS. Excellent agreement is seen for both the CDF and 95th percentile response.

\section{Conclusions}

In this paper, an assessment of various probabilistic methods for predicting the statistics of the forced response of mistuned bladed disks was carried out. Two blade vibration response cases were examined: (1) the response amplitude of a specific blade at a fixed frequency, and (2) the maximum response amplitude of any blade across the frequency range of interest.

For the first case, it was found that all the most probable point (MPP)-based methods considered- the first-order reliability method (FORM), second-order reliability method (SORM), and advanced mean value $(\mathrm{AMV}+)$ method-gave accurate and efficient predictions of the blade amplitude probability distributions, at least in limited ranges of uncertainties. However, for the second case, which is of primary engineering interest, the FORM, SORM, and AMV + methods all failed to predict the probabilistic response, regardless of the ranges of uncertainties. The MPP-based methods could not predict the probability for the second case because the performance function is much more nonlinear than a simple concave- or a convex-like function. It was also found that the response surface method (RSM) with 500 sampling points could not describe the 
actual structural response for the second case, although it did work for the first case. With more sampling points, the RSM could be used to describe the highly nonlinear response surface for the second case, but at the cost of a decrease in efficiency. Finally, the radius-based importance sampling method was shown to have poor efficiency for both response cases due to the large number of variables.

The performance of an accelerated Monte Carlo simulation method, which has been previously proposed for predicting the statistics of the maximum response of any blade over a frequency range, was also examined. This method is based on the property that the distribution of the maximum blade response amplitude asymptotically approaches the Weibull distribution (type III extreme value distribution). Thus, this approach is not appropriate for the first case, namely the response amplitude of a specific blade at a fixed frequency. However, for the second case, it allows for the construction of the Weibull distribution by running relatively few Monte Carlo realizations, and it was found to yield good predictions of the maximum blade amplitude statistics. Thus, this specialized probabilistic method clearly showed superior efficiency and accuracy compared to reliability-based methods for this important case of mistuned bladed disk vibration analysis.

\section{Acknowledgments}

This research was supported by the Automotive Research Center, a U.S. Army RDECOM center of excellence for modeling and simulation at the University of Michigan.

\section{References}

\footnotetext{
${ }^{1}$ Hodges, C. H., "Confinement Of Vibration By Structural Irregularity," Journal of Sound and Vibration, Vol. 82, No. 3, 1982, pp. 411-424.

${ }^{2}$ Hodges, C. H. and Woodhouse, J., "Vibration Isolation From Irregularity In A Nearly Periodic Structure: Theory and Measurements," Journal of Acoustical Society of America, Vol. 74, No. 3, 1983, pp. 894-905.

${ }^{3}$ Wei, S.-T. and Pierre, C., "Localization Phenomena in Mistuned Assemblies with Cyclic Symmetry Part II: Forced Vibrations," ASME Journal of Vibration, Acoustics, Stress, and Reliability in Design, Vol. 110, 1998, pp. 439-449.

${ }^{4}$ Sinha, A., "Calculating the Statistics of Forced Response of a Mistuned Bladed Disk Assembly," AIAA Journal, Vol. 24, No. 11, 1986, pp. 1797-1801.

${ }^{5}$ Griffin, J. H. and Hoosac, T. M., "Model Development and Statistical Investigation of Turbine Blade Mistuning," ASME Journal of Vibration, Acoustics, Stress, and Reliability in Design, Vol. 106, No. 2, 1984, pp. 204-210.

${ }^{6}$ Madsen, H. O., Krenk, S., and Lind, N. C., Methods of Structural Safety, Prentice-Hall, 1986.

${ }^{7}$ Breitung, K., "Asymptotic Approximations for Multinormal Integrals," Journal of Engineering Mechanics, Vol. 110, No. 3, 1984, pp. 357-366.

${ }^{8}$ Tvedt, L., "Distribution of Quadratic Forms in Normal Space-Application to Structural Reliability," Journal of Engineering Mechanics, Vol. 116, No. 6, 1990, pp. 1183-1197.

${ }^{9}$ Cai, G. Q. and Elishakoff, I., "Refined Second-Order Reliability Analysis," Structural Safety, Vol. 2, 1994, pp. 267-276.

${ }^{10}$ Madsen, H. O., "First Order vs. Second Order Reliability Analysis of Series Structures," Structural Safety, Vol. 2, 1985, pp. 207-214.

${ }^{11}$ Cizelj, L., Mavko, B., and Riesch-Oppermann, H., "Application of First and Second Order Reliability Methods in the Safety Assessment of Cracked Steam Generator Tubing," Nuclear Engineering and Design, Vol. 147, No. 3, 1994 , pp. 359-368.

${ }^{12} \mathrm{Wu}$, Y.-T., Millwater, H. R., and Cruse, T. A., "Advanced Probabilistic Structural Analysis Method for Implicit Performance Functions," AIAA Journal, Vol. 28, No. 9, 1990, pp. 1663-1669.

${ }^{13} \mathrm{Yu}$, X., Chang, K.-H., and Choi, K. K., "Probabilistic Structural Durability Prediction," AIAA Journal, Vol. 36, No. 4, 1998, pp. 628-637.

${ }^{14}$ Lee, S.-Y., Castanier, M. P., and Pierre, C., "Statistical Approximations of Power Flow for a Vehicle Structure with Uncertainties," Proceedings of ASME 2003 Design Engineering Technical Conference, ASME, Chicago, IL, 2003.

${ }^{15}$ Krishnamurthy, T., "Response Surface Approximation with Augmented and Compactly Supported Radial Basis Functions," Proceedings of the 44th AIAA/ASME/ASCE/AHS Structures, Structural Dynamics, and Material Conference and Exhibit, AIAA, Norfolk, VI, 2003.

${ }^{16}$ Belytschko, T., Lu, Y. Y., and Gu, L., "Element-Free Galerkin Methods," International Journal for Numerical Methods in Engineering, Vol. 37, 1994, pp. 229-256.

${ }^{17}$ Harbitz, A., "An Efficient Sampling Method for Probability of Failure Calculation," Structural Safety, Vol. 3, 1986, pp. 109-115.

${ }^{18}$ Castanier, M. P. and Pierre, C., "Consideration on the Benefits of Intentional Blade Mistuning for the Forced Response of Turbomachinery Rotors," Analysis and Design Issues for Modern Aerospace Vehicles, Vol. 55, 1997, pp. 419-425.

${ }^{19}$ Bladh, R., Pierre, C., Castanier, M.P., and Kruse, M.J., "Dynamic Response Predictions for a Mistuned Industrial Turbomachinery Rotor Using Reduced-Order Modeling," ASME Jourbnal of Engineering for Gas Turbines and Power, Vol. 124, 2002, pp. 311-324.

${ }^{20}$ Bendiksen, O. O., "Flutter of Mistuned Turbomachinery Rotors," ASME Jourbnal of Engineering for Gas Turbines and Power, Vol. 106, 1984, pp. 25-33.
} 
${ }^{21}$ Ottarson, G., and Pierre, C., "A Transfer Matrix Approach to Free Vibration Localization in Mistuned Bladed Assemblies," Journal of Sound and Vibration, Vol. 197, No. 5, 1996, pp. 589-618.

${ }^{22}$ Baik, S., Castanier, M.P., and Pierre, C., "Mistuning Sensitivity Prediction of Bladed Disks using Eigenvalue Curve Veerings," Proceedings of the 9th National Turbine Engin High Cycle Fatigue Conference, Pinehurst, NC, 2004.

${ }^{23}$ Hasofer, A. M. and Lind, N. C., "Exact and Invariant Second-Moment Code Format," Journal of Engineering Mechanics Division, ASCE, Vol. 100, No. 1, 1974, pp. 111-121.

${ }^{24}$ Tu, J., Choi, K. K., and Park, Y. H., "Design Potential Method for Robust System Parameter Design," AIAA Journal, Vol. 39, No. 4, 1998, pp. 667-677.

${ }^{25}$ Gumbel, E. J., Statistics of Extremes, Columbia University Press, New York, 1958.

${ }^{26}$ Whitehead, D. S., "The Maximum Factor by which Forced Vibration of Blades Can Increase due to Mistuning," ASME Jourbnal of Engineering for Gas Turbines and Power, Vol. 120, 1998, pp. 115-119. 